\title{
Correlates of externalising and internalising problems in children with dyslexia: An analysis of data from clinical casefiles
}

Mark E. Boyes ${ }^{1}$, Suze Leitão ${ }^{2}$, Mary Claessen $^{2}$, Nicholas A. Badcock $^{3}, \&$ Mandy Nayton ${ }^{4}$

1: School of Psychology, Faculty of Health Sciences, Curtin University, Perth, WA, Australia.

2: School of Occupational Therapy Social Work and Speech Pathology, Faculty of Health

Sciences, Curtin University, Perth, WA, Australia.

3: Department of Cognitive Science, Macquarie University, Sydney, NSW, Australia.

4: The Dyslexia-SPELD Foundation, Perth, WA, Australia.

\section{$\underline{\text { Citation }}$}

Boyes, M. E., Leitão, S., Claessen, M., Badcock, N. A., \& Nayton, M. (in press). Correlates of externalising and internalising problems in children with dyslexia: An analysis of data from clinical casefiles. Australian Psychologist. DOI: 10.1111/ap.12409.

\section{$\underline{\text { Acknowledgements }}$}

This work was supported by the Western Australian Health Promotion Foundation (Healthway), through research grant (24342). We would like to thank Sarah Holloway, Yppres Drew, and Kelly Fullarton for their assistance in data extraction and entry, and Tina Kilpatrick for her assistance in data coding.

NOTICE: this is the author's version of a work that was accepted for publication in Australian Psychologist. Changes resulting from the publishing process, e.g. peer review, editing, corrections, structural formatting, and other quality control mechanisms, may not be reflected in this document. Changes may have been made to this work since it was submitted to the journal. A definitive version was published in Australian Psychologist (DOI: 10.1111/ap.12409).

\section{Corresponding Author:}

Dr Mark Boyes

Senior Research Fellow

School of Psychology

Faculty of Health Sciences, Curtin University

GPO Box U1987

Perth, Western Australia, 6845

Phone: +61 892667025

Email: mark.boyes@curtin.edu.au

Web: http://markboyes.wix.com/markboyes

Twitter:@me_boyo 


\section{Abstract}

Objective: Adopting a socio-ecological perspective, this study used data extracted from clinical casefiles to investigate factors associated with externalising and internalising problems in a large, representative sample of children with a diagnosis of dyslexia. Method: This study is a secondary analysis of data collected by the Dyslexia-SPELD Foundation in Western Australia. Casefiles for school-aged children who had received a dyslexia diagnosis in 2014 and 2015 were identified $(n=1235)$, and a subset of casefiles were randomly selected for data extraction $(n=$ 454). Of the sample, $58 \%(n=262)$ were male, $42 \%(n=192)$ were female. Ages ranged between 6 and 17 years $(M=12.32, S D=3.07)$. Casefiles include results from assessments of literacy-related achievement, as well as parent-reported information on behavioural and socioemotional development. Results: After controlling for child age, gender, and reading ability, it was found that low self-esteem, difficulties in emotion regulation, and social skills difficulties were all associated with externalising problems. Additionally, low self-esteem, difficulties in emotion regulation, and bullying victimisation were all associated with internalising problems. Peer relationship difficulties were indirectly associated with both externalising and internalising problems via associations with low self-esteem, difficulties in emotion regulation, and social skills difficulties. Conclusion: Self-esteem, bullying victimisation, emotion regulation, social skills, and peer problems are salient correlates of externalising and internalising problems in children with a diagnosis of dyslexia. Implications for intervention are discussed, including both universal school-based mental health promotion programs, as well as more targeted programs for children with dyslexia.

Keywords: Dyslexia, Externalising, Internalising, Mental Health, Reading, Socio-ecological 


\section{Key Points}

\section{What is already known about this topic?}

1. Dyslexia is associated with elevated risk for both externalising and internalising problems; however, why dyslexia is associated with mental health difficulties remains unclear.

2. In the broader child psychology literature low self-esteem, peer relationships problems and bullying victimisation, difficulties in emotion regulation and coping, poor social skills, poor relationships with parents and family, academic failure, and poor school connectedness have been identified as correlates of child mental health problems.

3. Children with a diagnosis of dyslexia experience difficulties in many of these areas.

\section{What this paper adds?}

1. Self-esteem, bullying victimisation, emotion regulation, social skills, and peer problems are associated with externalising and internalising problems in children with dyslexia.

2. Peer relationship difficulties are indirectly associated with externalising and internalising problems, via associations with low self-esteem, difficulties in emotion regulation, and social skills difficulties.

3. Interventions targeting these factors, either in universal school-based programs or dyslexia-specific programs, might help prevent the development of externalising and internalising problems in children with dyslexia. 


\section{Correlates of externalising and internalising problems in children with dyslexia: An analysis of data from clinical casefiles}

It is generally accepted that approximately $5-10 \%$ of children have substantial difficulties in learning to read despite adequate cognitive abilities and classroom instruction (Snowling, 2008). This represents between one and three children in an average Australian classroom. A substantial body of literature has focused on cognitive factors that underlie reading difficulties, leading to the design and evaluation of interventions to improve reading (Bowers, Kirby, \& Deacon, 2010). Less research has focused on the potential psychological consequences associated with reading difficulties; however, a growing literature indicates that children with reading difficulties are at elevated risk for both externalising problems (disruptive behaviours, such as conduct problems and hyperactivity) and internalising problems (emotional symptoms, such as depression and anxiety; Maughan \& Carroll, 2006). While evidence for relationships between reading difficulties and poor mental health difficulties is accumulating, there is a dearth of information on why reading difficulties are associated with poor mental health outcomes. In order to design interventions to promote mental health in children with reading difficulties, it is essential to identify factors associated with psychological outcomes for these children (Boyes, Leitão, Claessen, Badcock, \& Nayton, 2016).

Given that disruptive behavioural symptoms are observable and create classroom difficulties, it is perhaps unsurprising that most research has focused on externalising problems. Reading difficulties have been associated with behavioural problems (Maughan \& Carroll, 2006; Snowling, Muter, \& Carroll, 2007), conduct disorder (Carroll, Maughan, Goodman, \& Meltzer, 2005), and both anger and aggression (Eissa, 2010; Morgan, Farkas, \& Wu, 2012). Less research has examined relationships between reading difficulties and internalising symptoms (Maughan \& Carroll, 2006); however, associations with depression (Arnold et al., 2005), anxiety (Arnold et al., 2005; Carroll et al., 2005), somatic complaints (Arnold et al., 
2005), and low mood (Carroll et al., 2005) have been reported. Importantly, longitudinal studies have demonstrated that reading difficulties predict later externalising symptoms (Halonen, Aunola, Ahonen, \& Nurmi, 2006; Snowling et al., 2007) and internalising symptoms (Arnold et al., 2005), indicating that reading difficulties are a risk factor for the development of mental health problems.

However, why reading difficulties are associated with mental health problems remains unclear. From the perspective of socio-ecological theory (e.g. Bronfenbrenner, 1979), a child is considered to be at the centre of a network of interacting influences operating at different levels (e.g. individual, family, and community). Importantly, these influences interact with each other and resources in one area may protect against deficits in another. Identifying factors associated with externalising and internalising problems in the context of reading ability could inform both the theoretical understanding of why children with reading difficulties are at elevated risk of mental health problems and also indicate potential factors to target in any intervention efforts (Boyes et al., 2016).

Little research has directly investigated risk/protective factors associated with externalising and internalising difficulties in children with reading difficulties; however, the broader child mental health literature offers some direction. Specifically, low self-esteem (Hammen \& Goodman-Brown, 1990; Mann, Hosman, Schaalma, \& de Vries, 2004), peer relationship problems and bullying victimisation (Arseneault, Bowes, \& Shakoor, 2010; DeaterDeckard, 2001), difficulties in emotion regulation (Carthy, Horesh, Apter, Edge, \& Gross, 2010; Silk, Steinberg, \& Morris, 2003), academic failure (Hinshaw, 1992; McLeod \& Fettes, 2007), and poor connection with school (Lester, Waters, \& Cross, 2013; Shochet, Dadds, Ham, \& Montague, 2006) have all been identified as important risk factors for the development of mental health problems in children and adolescents more generally. In contrast, good social skills (Segrin, 2000; Spence, 2003), an adaptive coping style and good emotion regulation skills (Compas, Connor-Smith, Saltzman, Harding Thomsen, \& Wadsworth, 2001; Silk et al., 2003), 
supportive parenting and good family relationships (Patterson, DeBaryshe, \& Ramsey, 1989; Repetti, Taylor, \& Seeman, 2002), and school connectedness (including positive relationships with teachers; Miller-Lewis et al., 2014; Shochet et al., 2006) have been demonstrated to be important protective factors. Children with reading problems may experience difficulties in many of these areas, including self-esteem (Alexander-Passe, 2006; Humphrey \& Mullins, 2002), peer problems and bullying victimisation (Ingesson, 2007; Leitão et al., 2017), as well as academic failure, poor school connectedness, and difficulties with teacher relationships (Gibson \& Kendall, 2010; Leitão et al., 2017).

However, research on mental health in the context of reading difficulties has a number of important limitations. First, although reading difficulties have been linked with internalising and externalising problems in both child and adolescent samples, studies are generally small, heterogeneous, and have limited age ranges. This heterogeneity makes it difficult to compare associations between reading difficulties and mental health across age, although for anxiety at least it appears that poor reader of all ages are at elevated risk (Francis, Caruana, Hudson, \& McArthur, 2019). Additionally, in the child psychology literature more broadly, there are gender differences in both externalising and internalising problems, with boys more likely to develop externalising difficulties and girls more likely to develop internalising difficulties (Leadbeater, Kuperminc, Blatt, \& Hertzog, 1999). Externalising symptoms also typically have an earlier onset than internalising symptoms (Costello, Mustillo, Erkanli, Keeler, \& Angold, 2003). Therefore, when investigating associations between reading difficulties and child mental health, it is important to consider potentially confounding effects of gender and age and adjust for these. To do this, larger and more representative samples are needed. Second, studies of mental health and potential risk/protective factors tend to be conducted in isolation. Few studies of internalising and externalising problems also measure risk/protective factors, and few studies of risk/protective factors include measures of mental health. In two notable exceptions, Terras and colleagues (2009) reported low self-esteem was correlated with social, emotional, and 
behavioural problems in a sample of children with dyslexia, and Boyes, Tebbutt, Preece, and Badcock (2018) demonstrated that self-esteem protected against negative impacts of poor reading ability on mental health outcomes. Further research assessing both child mental health and potential risk/protective factors in the same sample is needed.

Taken together, findings from the limited literature in the context of reading difficulties indicate that correlates of mental health in the general child psychology literature are likely good candidates when attempting to identify predictors of mental health in children with dyslexia. Indeed, given their importance for child mental health more generally, information regarding these factors is often routinely collected by clinicians providing assessment and treatment for dyslexia. However, additional literacy-related factors, such as the degree of reading impairment, family history of language or literacy difficulties, and whether a language other than English is spoken in the home, also need to be considered; either as important factors in their own right, or as potential confounders. This information is also routinely collected by clinical service providers. As such, collaborating with clinical service providers creates unique opportunities to use detailed clinical records to identify factors associated with mental health in children with dyslexia (Boyes et al., 2016).

The aim of the current exploratory study was to use data extracted from clinical casefiles to identify factors associated with externalising and internalising problems in a large representative sample of children who have received a diagnosis of dyslexia. Adopting socioecological theory (Bronfenbrenner, 1979) as an organisational framework onto which correlates could be mapped, hypothesised risk factors were organised into categories; child-related (low self-esteem, bullying victimisation, peer relationship difficulties, social skills difficulties, emotion regulation difficulties), family-related (difficulties in relationships with parents and siblings, parents separated or divorced), and school-related (perceived lack of school support, difficulties in relationships with teachers, academic failure). These risk factors were all predicted to be positively associated with externalising and internalising problems. Investigation 
of child (reading, spelling, and writing abilities) and family (family history of language or literacy problems and home language other than English) literacy-related factors was exploratory; however, it was predicted that poorer reading, spelling, and writing abilities, as well as family history of language or literacy difficulties, would be associated with more externalising and internalising problems.

\section{Method}

\section{Participants}

This study is a secondary analysis of data collected by the Dyslexia-SPELD Foundation (DSF) in 2014 and 2015. DSF has provided vital support to Western Australian children with reading and learning difficulties for 50 years and assesses over 1500 children and adolescents annually. The majority of families (over 95\%) have given consent for the data to be used for research purposes. As part of their clinical assessment, DSF creates a casefile for each child, including results from assessments of literacy-related achievement, as well as parent-reported information on behavioural and socio-emotional development. All casefiles for school-aged children who had received a dyslexia diagnosis from DSF in 2014 and 2015 were identified $(n=1235)$, and a subset of casefiles were randomly selected for data extraction $(n=454)$. In order to be diagnosed with dyslexia, DSF assessed students against the four diagnostic criteria specified in the DSM-5 (American Psychiatric Association, 2013). A diagnosis was made if students were: $i$ ) assessed as achieving substantially below their peers on standardised tests of literacy, with substantially below defined as greater than 1 standard deviation below their school-aged peers ${ }^{1}$, ii) were identified as failing to respond as expected to at least six months of targeted and explicit literacy intervention, iii) were currently of school age, and $i v$ ) were found not to present with

\footnotetext{
${ }^{1}$ Although it should be noted that, consistent with DSM 5 guidelines, a student may be deemed to meet criteria even if performing at levels close to or within the average range, if the student is receiving high levels of ongoing support and/or is contributing and sustaining unusually high levels of personal effort
} 
other disorders, adversities or environmental factors that could be viewed as more likely causative factors. Sample size was determined a priori based on a power analysis (conducted in GPower 3.0.10; Faul, Erdfelder, Lang, \& Buchner, 2007), which indicated that a sample of 139 was needed to detect a moderate effect (using $\alpha=.05$, power of .80 , and 15 predictors). To ensure adequate statistical power for analyses within different age categories (i.e. Primary School, Middle School, and Upper School) should this be needed in future, 150 casefiles were randomly selected from each, giving a total required sample of 450 casefiles. Of the sample, $58 \%(n=262)$ were male, $42 \%(n=192)$ were female, and ages ranged between 6 and 17 years $(M=12.32, S D=3.07)$

\section{Measures}

Reading and literacy: The Wechsler Individual Achievement Test, 2nd Edition (WIATII; The Psychological Corporation, 1992) was used to assess child reading (word reading and pseudoword reading), spelling, and written expression achievement. The WIAT-II is widely used and well validated, with excellent test re-test reliability $(r=0.85-0.98)$, internal consistency $(\alpha=0.80-0.98)$, and inter-rater reliability $(r=0.94-0.98)$, and has Australian age-standardised $(M=100, S D=15)$ norms (The Psychological Corporation, 1992; Weschler, 2002). Age-standardised scores are used in the current analyses.

The word reading task assesses pre-reading and decoding skills. The child is asked to complete the following activities; name the letters of the alphabet, identify and generate rhyming words, identify the beginning and ending sounds of words, match sounds with letters and letter blends, or read aloud a graded list of words. The starting point of this task is determined by students' year of school and the establishment of a basal ability level. The task is discontinued after 7 consecutive scores of 0 .

The pseudoword decoding task assesses the ability to apply phonetic decoding skills and involves children reading aloud a list of nonsense words designed to mimic the phonetic 
structure of real words in the English language. All students, regardless of age, start at item 1 and the task is discontinued after 7 consecutive scores of 0 .

The spelling task evaluates the child's spelling ability. This may include writing dictated letters, letter blends or words. The starting point of this task is determined by students' year of school and the establishment of a basal ability level. The task is discontinued after 6 consecutive scores of 0 .

The written expression task assesses the students' writing skills at all levels of language. This may include writing the alphabet, demonstrating written word fluency, combining and generating sentences or producing an extended writing piece (paragraph or persuasive essay). The starting point of this task is determined by students' year of school and discontinue rules are age dependent.

Externalising and internalising problems: The DSF parent questionnaire records information on child socio-emotional development. Externalising problems were captured with an open-ended item on any child "behaviour problems" which was dichotomised ( 0 : No behaviour problems mentioned; 1: Behaviour problems mentioned). Whilst there is not a specific question regarding emotional problems, there are a number of open-ended sections where parents are asked to provide details on anything that they believe would be helpful for the clinician to know about their child or family. This information was used to create a dichotomous internalising problems variable $(0$ : No internalising problems mentioned; 1 : Internalising problems mentioned). Internalising difficulties were recorded if any emotional symptoms (e.g. mood problems, depressive symptoms, anxiety symptoms, suicidal ideation, or posttraumatic stress, as well as related behaviours such as enuresis or toileting problems) were mentioned anywhere in the parent responses.

Hypothesized risk factors: All predicted risk factors were extracted from parent responses to the biographical information, speech and language development, educational history, and socio-emotional development sections of the DSF parent questionnaire. Responses 
were recorded as binary variables in the database, capturing whether the specific risk factor had been mentioned anywhere in the parent response ( 0 : Not mentioned; 1 : Mentioned). Childrelated factors included low self-esteem, bullying victimisation, difficulties with peer relationships, social skills difficulties, and difficulties in emotion-regulation. Family-related factors included speaking a home language other than English, a reported family history of speech language or literacy difficulties, parent-child relationship difficulties, difficulties in relationships with siblings, and whether the parents were divorced or separated. School-related factors included minimal or no school support for the diagnosis or additional needs, difficulties in teacher-child relationships, and frequent academic failure.

\section{Procedure}

All data were collected by DSF as part of standard diagnostic assessments and an opt-out consent procedure was implemented. Parents could indicate if they did not want their child's data to be used for research purposes. Data from children whose parents had chosen to opt out were not extracted or recorded in the database. Only de-identified data were made available to the researchers. Ethical approval for this secondary data analysis was obtained from the XXXX Human Research Ethics Committee (PSYSP 2014-76).

\section{Results}

Less than $2.1 \%$ of data were missing on externalising and internalising problems and their hypothesized correlates. These data were missing completely at random [Little's test: $\chi^{2}(252)=$ $255.65, p=.42]$ and the expectation maximization algorithm in SPSS 24 was used to impute missing values. Less than $2.7 \%$ of data were missing on the reading and literacy assessments and although these data were not missing completely at random [Little's test: $\chi^{2}(10)=25.14, p=$ 
$.01]$, given the minimal amount of missing data, expectation maximisation was again used to impute missing values.

\section{Descriptive statistics and bivariate analyses}

The proportion of children for whom parents indicated externalising and internalising problems was estimated and descriptive statistics were calculated, disaggregated by externalising and internalising problems. The mean age of children in the sample was 12.32 years $(S D=3.07)$. There were significantly more males than females in the sample [Male: $n=262,58 \%$; Female: $n$ $\left.=192,42 \% ; \chi^{2}(1)=10.79, p<0.001\right]$. Overall, children scored approximately one standard deviation below the age-normed means on the standardised reading and literacy measures (Word Reading: $M=86.85, S D=14.54$; Pseudoword Reading: $M=85.69, S D=13.33$; Spelling: $M=$ 85.03, $S D=11.43$; Written Expression: $M=86.53, S D=12.65)$. Parents reported externalising problems for $24 \%(n=111)$ and internalising problems for $9 \%(n=39)$ of children.

One-way ANOVAs and chi-squared tests were conducted to examine bivariate associations between literacy scores on the WIAT-II, hypothesised risk factors, and parentreported externalising and internalising problems. There were no significant differences in age, gender, and reading/literacy scores as function of externalising or internalising problems. In bivariate analyses, in comparison with children with no externalising and/or internalising problems, children with externalising/internalising problems were significantly more likely to have low self-esteem [Externalising: $\chi^{2}(1)=51.51, p<0.001$; Internalising: $\chi^{2}(1)=8.77, p<$ 0.001], difficulties in peer relationships [Externalising: $\chi^{2}(1)=28.03, p<0.001$; Internalising: $\chi^{2}(1)=4.43, p=0.04$ ], and difficulties in emotion regulation [Externalising: $\chi^{2}(1)=87.54, p<$ 0.001; Internalising: $\chi^{2}(1)=5.68, p=0.02$ ]. Additionally, children with externalising problems were more likely to have social skills difficulties $\left[\chi^{2}(1)=14.97, p<0.001\right]$ than children with no externalising problems, and children with internalising problems were more likely to have experienced bullying victimisation $\left[\chi^{2}(1)=15.17, p<0.001\right]$ than children with no internalising 
problems. Difficulties in parent $\left[\chi^{2}(1)=4.07, p=0.04\right]$, sibling $\left[\chi^{2}(1)=12.90, p<0.001\right]$, and teacher relationships $\left[\chi^{2}(1)=9.19, p=0.01\right]$ were all associated with externalising problems. None of the family or school related factors were associated with internalising problems in bivariate analyses.

\section{(Insert Table 1 approximately here)}

\section{Multivariate analyses}

Two multiple logistic regressions were conducted to determine whether the hypothesised risk factors were independently associated with externalising and internalising problems, after controlling for child age, gender, and literacy scores. The overall model for externalising problems was significant $\left[\chi^{2}(17)=136.45, p<0.001\right]$ and accounted for $26 \%-39 \%$ of the variance [Cox \& Snell $R^{2}=.26$; Nagelkerke $\left.R^{2}=.39\right]$. Low self-esteem $(O R=3.69)$, social skills difficulties $(O R=4.94)$, and difficulties in emotion regulation $(O R=14.27)$ were the only risk factors significantly associated with externalising problems (Table 2). The overall model for internalising problems was also significant $\left[\chi^{2}(17)=27.51, p=0.05\right]$ and accounted for $6 \%-$ $13 \%$ of the total variance [Cox \& Snell $R^{2}=0.06$; Nagelkerke $R^{2}=0.13$ ]. Low self-esteem $(O R$ $=2.31)$, bullying victimisation $(O R=4.33)$, and difficulties in emotion regulation $(O R=2.45)$ were the only risk factors significantly associated with internalising problems (Table 2).

\section{(Insert Table 2 approximately here)}

Peer and teacher relationship difficulties: Indirect associations with externalising and internalising problems

Although significantly associated with both externalising and internalising problems in bivariate analyses, peer relationship difficulties were not significantly associated with externalising and 
internalising problems in the multivariate analyses. This raised the possibility that peer relationship problems may be indirectly associated with externalising and internalising problems via associations with low self-esteem, difficulties in emotion regulation, and bullying victimisation. Exploratory tests of multiple mediation were conducted to test this possibility. Analyses were conducted in Mplus 6 using the WLSMV estimator. Estimates and bias-corrected 95\% confidence intervals were generated using 1000 bootstrapped resampling draws. Unstandardised estimates are reported, as recommended by Hayes (2013). Indirect effects were determined to be significant if the confidence interval for the estimate did not overlap zero (Hayes, 2013).

Externalising problems: Consistent with the results of the logistic regression there was no direct effect of peer relationship difficulties $(B=-0.53,95 \% \mathrm{CI}=-1.25-0.17)$ on externalising problems. However, the total indirect effect of peer relationship difficulties was significant $(B=1.35,95 \% \mathrm{CI}=0.58-1.96)$. More specifically, the indirect effects operating through associations with low self-esteem $(B=0.29,95 \% \mathrm{CI}=0.11-0.53)$ and difficulties in emotion regulation $(B=0.57,95 \% \mathrm{CI}=0.26-0.87)$ were both significant (Figure 1). The indirect effect operating through associations with social skills difficulties was not significant ( $B$ $=0.49,95 \% \mathrm{CI}=-0.06-1.16)$.

\section{(Insert Figure 1 approximately here)}

Internalising problems: Consistent with the results of the logistic regression there was no direct effect of peer relationship difficulties $(B=-0.28,95 \% \mathrm{CI}=-1.03-0.34)$ on internalising problems. However, the total indirect effect of peer relationship difficulties was significant $(B=$ $0.71,95 \% \mathrm{CI}=0.29-1.14)$. More specifically, the indirect effects operating through associations with low self-esteem $(B=0.21,95 \% \mathrm{CI}=0.04-0.46)$ and difficulties in emotion regulation $(B=0.22,95 \% \mathrm{CI}=0.00-0.50)$ were significant (Figure 2$)$. The indirect effect 
operating through associations with bullying victimisation was not significant $(B=0.28,95 \% \mathrm{CI}$ $=-0.01-0.63)$, even though the relevant direct associations were all significant.

\section{(Insert Figure 2 approximately here)}

\section{Discussion}

Adopting a socio-ecological framework (Bronfenbrenner, 1979), the current study aimed to explore child, family, and school-related factors associated with externalising and internalising problems in children with a diagnosis of dyslexia. Following recent recommendations (Boyes et al., 2016), an innovative aspect of the study was the use of data routinely collected by clinical service providers. Low self-esteem and difficulties in emotion regulation were associated with parent-reported externalising and internalising problems. Additionally, poor social skills were associated with externalising problems and bullying victimisation was associated with internalising problems. Although not directly associated with either externalising or internalising problems, peer relationship difficulties were indirectly associated with both, through associations with low self-esteem, difficulties in emotion regulation, and social skills difficulties. These findings are consistent with the broader child mental health literature, as well as the themes raised in qualitative interviews with both children with dyslexia and their parents (Leitão et al., 2017). Findings indicate that addressing self-esteem, emotion-regulation, social skills, and peer relationships may promote mental health in children with a diagnosis of dyslexia. Surprisingly, none of the family- or school-related factors were associated with externalising or internalising problems in multivariate analyses, although difficulties in parent, sibling, and teacher relationships were associated with externalising problems in univariate analyses. 
Although the current findings highlight the utility of collaborating with clinical service providers, and offer potential implications for intervention, the results need to be considered in light of some methodological limitations and constraints of the dataset. First, the cross-sectional nature of the data precludes any conclusions regarding the direction of associations observed. Longitudinal research is clearly needed to address this, particularly as relationships between risk factors (e.g. bullying victimisation) and mental health outcomes may be bi-directional (Boyes, Cluver, Bowes, Ward, \& Badcock, 2014). For example, tracing and reassessing the children included in the database would allow the predictive utility of the risk factors identified in the current study to be assessed. Additionally, conducting training or treatment studies and assessing changes in mental health would be a first step in attempting to establish causality. Second, the lack of a comparison group without a diagnosis of dyslexia means that it is not possible to determine whether dyslexia was associated with elevated externalising and internalising problems in the current sample, or whether the risk factors identified moderate and/or mediate potential associations between dyslexia and psychological problems. This needs to be addressed in future research.

Finally, and most importantly, only information from parents was available, and as the information on socio-emotional and behavioural development was collected as part of a much broader interview and assessment protocol, information on externalising and internalising problems, as well as potential risk factors, was limited to single items or responses to openended questions. Further research using reliable and valid measures of externalising and internalising problems, as well as their correlates, is clearly needed. Reliably measuring externalising and internalising symptoms in children is difficult and symptoms may vary across contexts (De Los Reyes \& Kazdin, 2005). Therefore, it will also be important to supplement parent report with child and teacher report, and triangulate these data where possible. Potentially, parent and teacher reports may offer more accurate estimates of externalising problems, as social desirability may result in children underreporting behaviour issues. In 
contrast, children may be better placed to report on their own unobservable and subjective affective state. Many validated measures of child socio-emotional functioning (such as the Strengths and Difficulties Questionnaire, Goodman, 1997) are short, easy to administer, provide an index of both externalising and internalising difficulties, and can be completed by children, parents, and/or teachers. Clinical service providers could consider including such measures in routine assessments.

Bearing these limitations in mind, from an intervention perspective, many universal school-based mental health promotion programs focus on competence, resilience, and skill building (e.g. emotion recognition and regulation, social skills, positive thinking, and social/interpersonal skills; Roberts et al., 2010) and have been demonstrated to be effective. Relatedly, a systematic review (Vreeman \& Carroll, 2007) and meta-analysis (Ttofi \& Farrington, 2011) of anti-bullying interventions both concluded that anti-bullying programs are effective in reducing bullying and victimisation, and the chances of success are greater when interventions incorporate a whole-school approach involving multiple disciplines and the whole school community (Vreeman \& Carroll, 2007). However, universal mental health or prevention programs typically yield small effect sizes (Durlak, Weissberg, Dymnicki, Taylor, \& Schellinger, 2011). This is perhaps unsurprising, as the majority of school children will not develop a mental health problem. Given this, future research could investigate whether such programs might be particularly effective for potentially vulnerable subgroups, such as children with a dyslexia diagnosis.

However, many universal mental health promotion programs place demands on the literacy skills of participants, making them less accessible to participants with dyslexia. Therefore, dyslexia-specific interventions, which target these same socio-emotional skills but are explicitly framed around the experience of reading difficulties and consider the literacy skills of participants in the design of program materials, should also be developed and trialled. This may be particularly important in the context of stressful periods, such as the transition from 
primary to secondary school (Bailey \& Baines, 2012; Eissa, 2010; Maguire \& Yu, 2015; See \& Gorard, 2014). Little research has examined the impact of interventions aiming to improve psychosocial health in children with dyslexia specifically. Firth and Frydenberg (2011) developed a coping program for children with learning difficulties. The program is yet to be tested in rigorous randomised trials, although there is preliminary evidence that it is associated with increases in adaptive coping and perceptions of control (Firth, Frydenberg, \& Greaves, 2010; Firth, Frydenberg, Steeg, \& Bond, 2013). Additionally, measures of internalising and externalising symptomatology are yet to be administered in any evaluations of the program, so whether program participation translates into improvements in child mental health is unknown. Further evaluation of existing programs, and development of new programs as evidence for risk/protective factors accumulates, may be warranted.

The current findings indicate that self-esteem, bullying victimisation, emotion regulation, social skills, and peer problems are all associated with externalising and internalising problems in children with a diagnosis of dyslexia. Interventions targeting these socio-emotional factors, either in universal school-based programs or dyslexia-specific programs, may prevent the development of externalising and internalising problems in children with dyslexia. 


\section{References}

Alexander-Passe, N. (2006). How dyslexic teenagers cope: An investigation of self-esteem, coping and depression. Dyslexia, 12, 26-275. doi:10.1002/dys.318

American Psychiatric Association. (2013). Diagnostic and statistical manual of the mental disorders (5th Edition). Arlington: American Psychiatric Association.

Arnold, E., Goldston, D., Walsh, A., Reboussin, B., Daniel, S., Hickman, E., \& Wood, F. (2005). Severity of emotional and behavioral problems among poor and typical readers. Journal of Abnormal Child Psychology, 33, 205-217. doi:10.1007/s10802-005-1828-9

Arseneault, L., Bowes, L., \& Shakoor, S. (2010). Bullying victimization in youths and mental health problems: 'Much ado about nothing'? Psychological Medicine, 40, 717-729. doi:10.1017/S0033291709991383

Bailey, S., \& Baines, E. (2012). The impact of risk and resiliency factors on the adjustment of children after the transition from primary to secondary school. Educational and Child Psychology, 29, 47-63.

Bowers, P. N., Kirby, J. R., \& Deacon, H. (2010). The effects of morphological instruction on literacy skills: A systematic review of the literature. Review of Educational Research, 80, 144-179. doi: 10.3102/0034654309359353

Boyes, M. E., Cluver, L., Bowes, L., Ward, C., \& Badcock, N. (2014). Bullying victimisation, internalising symptoms, and conduct problems in South African children and adolescents: A longitudinal investigation. Journal of Abnormal Child Psychology, 42, 1313-1324. doi:10.1007/s10802-014-9888-3

Boyes, M. E., Leitão, S., Claessen, M., Badcock, N. A., \& Nayton, M. (2016). Why are reading difficulties associated with mental health problems? Dyslexia, 22, 263-266. doi:10.1002/dys.1531 
Boyes, M. E., Tebbutt, B., Preece, K., \& Badcock, N. A. (2018). Relationships between reading ability and child mental health: Moderating effects of self-esteem. Australian Psychologist, 53, 125-133. doi:10.1111/ap.12281

Bronfenbrenner, U. (1979). The ecology of human development: Experiments by nature and design. Cambridge: Harvard University Press.

Carroll, J. M., Maughan, B., Goodman, R., \& Meltzer, H. (2005). Literacy difficulties and psychiatric disorders: Evidence for comorbidity. Journal of Child Psychology and Psychiatry, 46, 524-532. doi:10.1111/j.1469-7610.2004.00366.x

Carthy, T., Horesh, N., Apter, A., Edge, M. D., \& Gross, J. J. (2010). Emotional reactivity and cognitive regulation in anxious children. Behaviour Research and Therapy, 48, 384-393. doi:10.1016/j.brat.2009.12.013

Compas, B. E., Connor-Smith, J. K., Saltzman, H., Harding Thomsen, A., \& Wadsworth, M. E. (2001). Coping with stress during childhood and adolescence: Problems, progress, and potential in theory and research. Psychological Bulletin, 127, 87-127. doi:10.1037//00332909.127

Costello, E., Mustillo, S., Erkanli, A., Keeler, G., \& Angold, A. (2003). Prevalence and development of psychiatric disorders in childhood and adolescence. Archives of General Psychiatry, 60, 837-844. doi:10.1001/archpsyc.60.8.837

De Los Reyes, A., \& Kazdin, A. E. (2005). Informant discrepancies in the assessment of childhood psychopathology: A critical review, theoretical framework, and recommendations for further study. Psychological Bulletin, 131, 483-509. doi:10.1037/0033-2909.131.4.483

Deater-Deckard, K. (2001). Annotation: Recent research examining the role of peer relationships in the development of psychopathology. Journal of Child Psychology and Psychiatry, 42, 565-579. doi:10.1111/1469-7610.00753 
Durlak, J. A., Weissberg, R. P., Dymnicki, A. B., Taylor, R. D., \& Schellinger, K. B. (2011). The impact of enhancing students' social and emotional learning: A meta-analysis of school-based universal interventions. Child Development, 82, 405-432. doi:10.1111/j.1467-8624.2010.01564.x

Eissa, M. (2010). Behavioral and emotional problems associated with dyslexia in adolescence. Current Psychiatry, 17, 17-25.

Faul, F., Erdfelder, E., Lang, A.-G., \& Buchner, A. (2007). G*Power 3: A flexible statistical power analysis program for social, behavioral, and biomedical sciences. Behavior Research Methods, 39, 175-191. doi:10.3758/BF03193146

Firth, N., \& Frydenberg, E. (2011). Success and dyslexia: sessions for coping in the upper primary years. Camberwell: Australian Council of Educational Research.

Firth, N., Frydenberg, E., \& Greaves, D. (2010). Perceived control and adaptive coping: programs for adolescent students who have learning disabilities. Learning Disabilities Quarterly, 31, 151-165. doi:10.2307/25474645

Firth, N., Frydenberg, E., Steeg, C., \& Bond, L. (2013). Coping successfully with dyslexia: an initial study of an inclusive school-based resilience programme. Dyslexia, 19, 113-130. doi:10.1002/dys.1453

Francis, D., Caruana, N., Hudson, J., \& McArthur, G. (2019). The association between poor reading and internalising problems: a systematic review and meta-analysis. Clinical Psychology Review, 67, 45-60. doi:10.1016/j.cpr.2018.09.002

Gibson, S., \& Kendall, L. (2010). Stories from school: Dyslexia and learners' voices on factors impacting on achievement. Support for Learning, 25, 187-193. doi:10.1111/j.14679604.2010.01465.x

Halonen, A., Aunola, K., Ahonen, T., \& Nurmi, J.-E. (2006). The role of learning to read in the development of problem behaviour: A cross-lagged longitudinal study. British Journal of Educational Psychology, 76, 517-534. doi:10.1348/000709905X51590 
Hammen, C., \& Goodman-Brown, R. (1990). Self schemes and vulnerability in specific life stress in children at risk for depression. Cognitive Therapy and Research, 14, 215-227. doi:10.1007/BF01176210

Hayes, A. F. (2013). Introduction to mediation, moderation, and conditional process analysis. New York: Guilford Press.

Hinshaw, S. P. (1992). Externalizing behavior problems and academic underachievement in childhood and adolescence: Causal relationships and underlying mechanisms. Psychological Bulletin, 111, 127-155. doi:10.1037/0033-2909.111.1.127

Humphrey, N., \& Mullins, P. M. (2002). Self-concept and self-esteem in developmental dyslexia. Journal of Research in Special Educational Needs, 2. doi:10.1111/j.14713802.2002.00163.x

Ingesson, S. G. (2007). Growing up with dyslexia: Interviews with teenagers and young adults. School Psychology International, 28, 574-591. doi:10.1177/0143034307085659

Leadbeater, B., Kuperminc, G., Blatt, S., \& Hertzog, C. (1999). A multivariate model of gender differences in adolescents internalizing and externalizing problems. Developmental Psychology, 35, 1268-1282. doi:10.1037/0012-1649.35.5.1268

Leitão, S., Dzidic, P., Claessen, M., Gordon, J., Howard, K., Nayton, M., \& Boyes, M. E. (2017). Exploring the impact of living with dyslexia: The perspectives of children and their parents. International Journal of Speech-Language Pathology, 19, 322-334. doi:10.1080/17549507.2017.1309068

Lester, L., Waters, S., \& Cross, D. (2013). The relationship between school connectedness and mental health during the transition to secondary school: A path analysis. Australian Journal of Guidance and Counselling, 23, 157-171. doi:10.1017/jgc.2013.20

Maguire, B., \& Yu, M. (2015). Transition to secondary school The Longitudinal Study of Australian Children Annual statistical report 2014. Melbourne: Australian Institute of Family Studies. 
Mann, M., Hosman, C., Schaalma, H., \& de Vries, N. (2004). Self-esteem in a broad-spectrum approach for mental health promotion. Health Education Research, 19, 357-372. doi:10.1093/her/cyg041

Maughan, B., \& Carroll, J. M. (2006). Literacy and mental disorders. Current Opinion in Psychiatry, 19, 350-355. doi:10.1097/01.yco.0000228752.79990.41

McLeod, J. D., \& Fettes, D. L. (2007). Trajectories of failure: The educational careers of children with mental health problems. American Journal of Sociology, 113, 653-701. doi:10.1086/521849

Miller-Lewis, L. R., Sawyer, A. C. P., Searle, A. K., Mittinty, M. N., Sawyer, M. G., \& Lynch, J. W. (2014). Student-teacher relationship trajectories and mental health problems in young children. BMC Psychology, 2, 27. doi:10.1186/s40359-014-0027-2

Morgan, P., Farkas, G., \& Wu, Q. (2012). Do poor readers feel angry, sad, and unpopular? Scientific Studies of Reading, 16, 360-381. doi:10.1080/10888438.2011.570397

Patterson, G. R., DeBaryshe, B., \& Ramsey, E. (1989). A developmental perspective on antisocial behaviour. American Psychologist, 44, 329-335. doi:10.1037/0003066X.44.2.329

Repetti, R. L., Taylor, S. E., \& Seeman, T. E. (2002). Risky families: Family social environment $\mathrm{s}$ and the mental and physical health of offspring. Psychological Bulletin, 128, 330-366. doi:10.1037//0033-2909.128.2.330

Roberts, C., Kane, R. T., Bishop, B., Cross, D., Fenton, J., \& Hart, B. (2010). The prevention of anxiety and depression in children from disadvantaged schools. Behaviour Research and Therapy, 48, 68-73. doi:10.1016/j.brat.2009.09.002

See, B. H., \& Gorard, S. (2014). Improving literacy in the transition period : A review of the existing evidence on what works. British Journal of Education Society and Behavioural Science, 4, 739-754. 
Segrin, C. (2000). Social skills deficits associated with depression. Clinical Psychology Review, 20, 379-403. doi:10.1016/S0272-7358(98)00104-4

Shochet, I., Dadds, M., Ham, D., \& Montague, R. (2006). School connectedness is an underemphasized parameter in adolescent mental health: Results of a community prediction study. Journal of Clinical Child and Adolescent Psychology, 35, 170-179. doi:10.1207/s15374424jccp3502_1

Silk, J., Steinberg, L., \& Morris, A. (2003). Adolescents' emotion regulation in daily life: Links to depressive symptoms and problem behavior. Child Development, 74, 1869-1880. doi:10.1046/j.1467-8624.2003.00643.x

Snowling, M. J. (2008). State-of-Science Review: SR-D2: Dyslexia. Retrieved from London: Office for Science:

Snowling, M. J., Muter, V., \& Carroll, J. M. (2007). Children at family risk of dyslexia: A follow-up in early adolescence. Journal of Child Psychology and Psychiatry, 48, 609618. doi:10.1111/j.1469-7610.2006.01725.x

Spence, S. (2003). Social skills training with children and young people: Theory, evidence and practice. Child and Adolescent Mental Health, 8, 84-96. doi:10.1111/1475-3588.00051

Terras, M., Thompson, L., \& Minnis, H. (2009). Dyslexia and psycho-social functioning: An exploratory study of the role of self-esteem and understanding. Dyslexia, 15, 304-327. doi:10.1002/dys.386

The Psychological Corporation. (1992). Weschler Individual Achievement Test-2nd Edition. San Antonio: The Psychological Corporation.

Ttofi, M. M., \& Farrington, D. P. (2011). Effectiveness of school-based programs to reduce bullying: a systematic and meta-analytic review. Journal of Experimental Criminology, 7, 27-56. doi: 10.1007/s11292-010-9109-1 
Dyslexia and child mental health 25

Vreeman, R. C., \& Carroll, A. E. (2007). A systematic review of school-based interventions to prevent bullying. Archives of Pediatric and Adolescent Medicine, 161, 78-88. doi: 10.1001/archpedi.161.1.78

Weschler, D. (2002). Wechsler Individual Achievement Test - Second Edition: Australian Standardised Edition. Sydney: PsychCorp. 
Table 1. Descriptive statistics disaggregated by externalising and internalising problems

Externalising Problems

No $(n=343,76 \%) \quad$ Yes $(n=111,24 \%) \quad p$
Internalising Problems

No $(n=415,91 \%) \quad$ Yes $(n=39,9 \%) \quad p$

\section{Reading and Literacy}

WIAT - Word Reading $(M, S D)$

$87.31(14.28)$

$85.44(15.27)$

0.24

$86.62(14.76)$

$89.36(11.84)$

0.26

WIAT - Pseudoword Decoding $(M, S D)$

85.92 (12.93)

$84.97(14.53)$

0.51

$85.53(12.95)$

$87.38(16.99)$

0.41

WIAT - Spelling $(M, S D)$

85.34 (11.39)

84.09 (11.56)

$0.32 \quad 84.94(11.50)$

$86.00(10.74)$

0.58

$87.06(12.45)$

$84.91(13.17)$

0.12

$86.23(12.51)$

$89.77(13.82)$

0.09

\section{Child-Related Factors}

Age $(M, S D)$

Peer-Child Relationship Difficulties ( $n, \%)$
$12.41(3.05)$

$12.03(3.13)$

0.25

12.33 (3.09)

$0.67 \quad 174(42 \%)$

$<\mathbf{0 . 0 0 1} 101(24 \%)$

$0.1520(5 \%)$

$<\mathbf{0 . 0 0 1} 63(15 \%)$

$36(32 \%)$

$38(11 \%)$

$10(9 \%)$

$<\mathbf{0 . 0 0 1} 13(3 \%)$

$12.16(2.86)$

0.74

$5(1.5 \%)$
$18(46 \%)$

$18(46 \%)$

$8(21 \%)$

$11(28 \%)$

$2(5 \%)$
0.61

0.01

$<0.001$

0.04

0.51 


\section{Family-Related Factors}

\begin{tabular}{|c|c|c|c|c|c|c|}
\hline Home Language Other Than English $(n, \%)$ & $27(8 \%)$ & $8(7 \%)$ & 0.82 & $33(8 \%)$ & $2(5 \%)$ & 0.53 \\
\hline Family History of Language Difficulties $(n, \%)$ & $13(4 \%)$ & $9(8 \%)$ & 0.07 & $21(5 \%)$ & $1(2 \%)$ & 0.49 \\
\hline Parent-Child Relationship Difficulties ( $n, \%)$ & $14(4 \%)$ & $10(9 \%)$ & 0.04 & $20(5 \%)$ & $4(10 \%)$ & 0.15 \\
\hline Sibling-Child Relationship Difficulties ( $n, \%)$ & $12(4 \%)$ & $14(13 \%)$ & $<0.001$ & $24(6 \%)$ & $2(5 \%)$ & 0.87 \\
\hline Parents Divorced/Separated $(n, \%)$ & $16(5 \%)$ & $10(9 \%)$ & 0.09 & $24(6 \%)$ & $25 \%)$ & 0.87 \\
\hline \multicolumn{7}{|l|}{ School-Related Factors } \\
\hline Lack of School Support $(n, \%)$ & $10(3 \%)$ & $5(5 \%)$ & 0.42 & $13(3 \%)$ & $2(5 \%)$ & 0.51 \\
\hline Teacher-Child Relationship Problems $(n, \%)$ & $38(11 \%)$ & $25(23 \%)$ & 0.01 & $56(14 \%)$ & $7(18 \%)$ & 0.44 \\
\hline Academic Failure $(n, \%)$ & $30(9 \%)$ & $12(11 \%)$ & 0.51 & $38(9 \%)$ & $4(10 \%)$ & 0.82 \\
\hline
\end{tabular}

Note. Significance tests are associated with one-way ANOVAs and chi squared tests. Significant $p$ values are bolded. Percentages are rounded to nearest whole percent. 
Table 2. Summary of multiple logistic regression models for externalising and internalising problems

Externalising Problems

$B(\mathrm{SE})$

OR $(95 \% \mathrm{CI})$

$p$

Internalising Problem

\section{Child-Related Factors}

Low Self-esteem

Bullying Victimisation

Peer-Child Relationship Difficulties

Social Skills Difficulties

Emotion-Regulation Difficulties

\section{Family-Related Factors}

Home Language Other Than English

Family History of Language Difficulties

Parent-Child Relationship Difficulties

Sibling-Child Relationship Difficulties

Parents Divorced/Separated

$\begin{array}{llllll}1.30(0.30) & 3.69(2.06-6.60) & \mathbf{< 0 . 0 0 1} & 0.84(0.39) & 2.31(1.07-4.90) & \mathbf{0 . 0 3} \\ -0.58(0.54) & 0.56(0.20-1.60) & 0.28 & 1.47(0.54) & 4.33(1.50-12.49) & \mathbf{0 . 0 1} \\ 0.66(0.35) & 1.93(0.96-3.86) & 0.06 & 0.31(0.46) & 1.35(0.56-3.33) & 0.50 \\ 1.60(0.66) & 4.94(1.34-18.15) & \mathbf{0 . 0 2} & -0.28(0.88) & .76(0.14-4.26) & 0.75 \\ 2.66(0.39) & 14.27(6.61-30.81) & \mathbf{< 0 . 0 0 1} & 0.90(0.46) & 2.45(1.00-6.03) & \mathbf{0 . 0 5}\end{array}$

$\begin{array}{llllll}-0.49(0.56) & 0.61(0.21-1.82) & 0.38 & -0.81(0.81) & 0.45(0.09-2.19) & 0.32 \\ 1.01(0.56) & 2.74(0.91-8.26) & 0.07 & -0.84(1.10) & 0.43(0.05-3.69) & 0.44 \\ 0.66(0.54) & 1.93(0.66-5.56) & 0.23 & 0.63(0.69) & 1.87(0.49-7.18) & 0.36 \\ 0.87(0.51) & 2.38(0.88-6.43) & 0.09 & -0.66(0.85) & 0.52(0.10-2.72) & 0.44 \\ -0.01(0.53) & 1.00(0.36-2.79) & 0.99 & -0.86(0.85) & 0.42(0.08-2.23) & 0.31\end{array}$




\section{School-Related Factors}

Lack of School Support

$0.76(0.67)$

$2.13(0.58-7.89)$

0.26

$0.12(0.88) \quad 1.13(0.20-6.37)$

0.89

Teacher-Child Relationship Problems

$0.44(0.37)$

$1.55(0.76-3.19)$

0.23

$-0.01(0.51) \quad 0.99(0.37-2.69)$

0.99

Academic Failure

$-0.16(0.44)$

$0.86(0.36-2.02)$

0.72

$-0.40(0.63) \quad 0.67(0.20-2.33)$

0.53

Note. $\mathrm{SE}=$ Standard Error. $\mathrm{OR}=$ Odds Ratio. $\mathrm{CI}=$ Confidence Interval. Significant $p$ values are bolded. Models control for child age, gender, and literacy scores (WIAT-II). 
Figure 1. Indirect effects of difficulties in peer relationships on externalising problems, operating via associations with low self-esteem, difficulties in emotion regulation, and social skills difficulties (Note: $* * * p<.001, * * p<.01, * p<.05$. Unstandardised coefficients are reported. Dashed lines indicate a non-significant association)

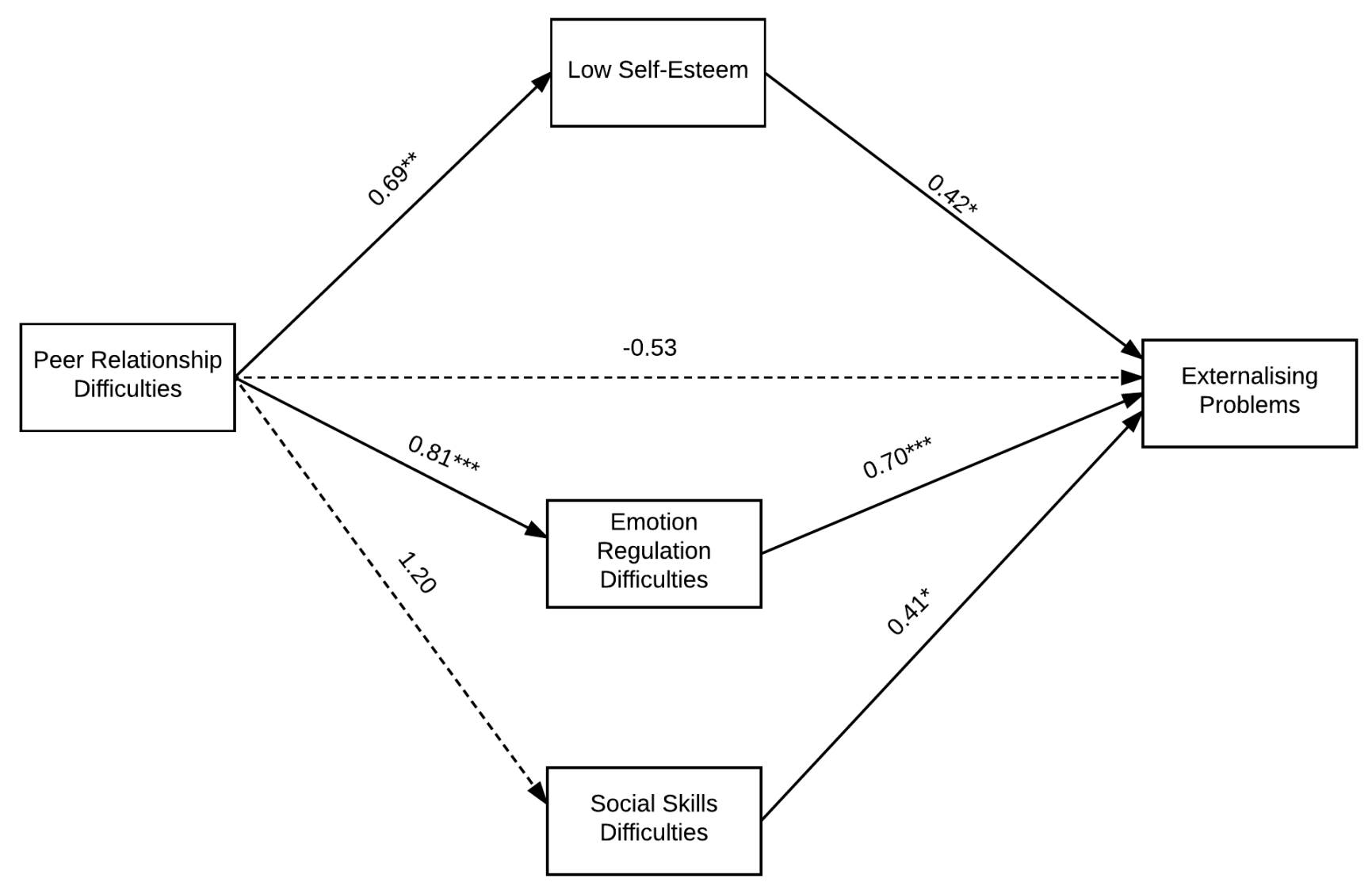


Figure 2. Indirect effects of difficulties in peer relationships on internalising problems, operating via associations with low self-esteem,

difficulties in emotion regulation, and bullying victimisation (Note: $* * * p<.001,{ }^{* *} p<.01, * p<.05$. Unstandardised coefficients are reported.

Dashed lines indicate a non-significant association)

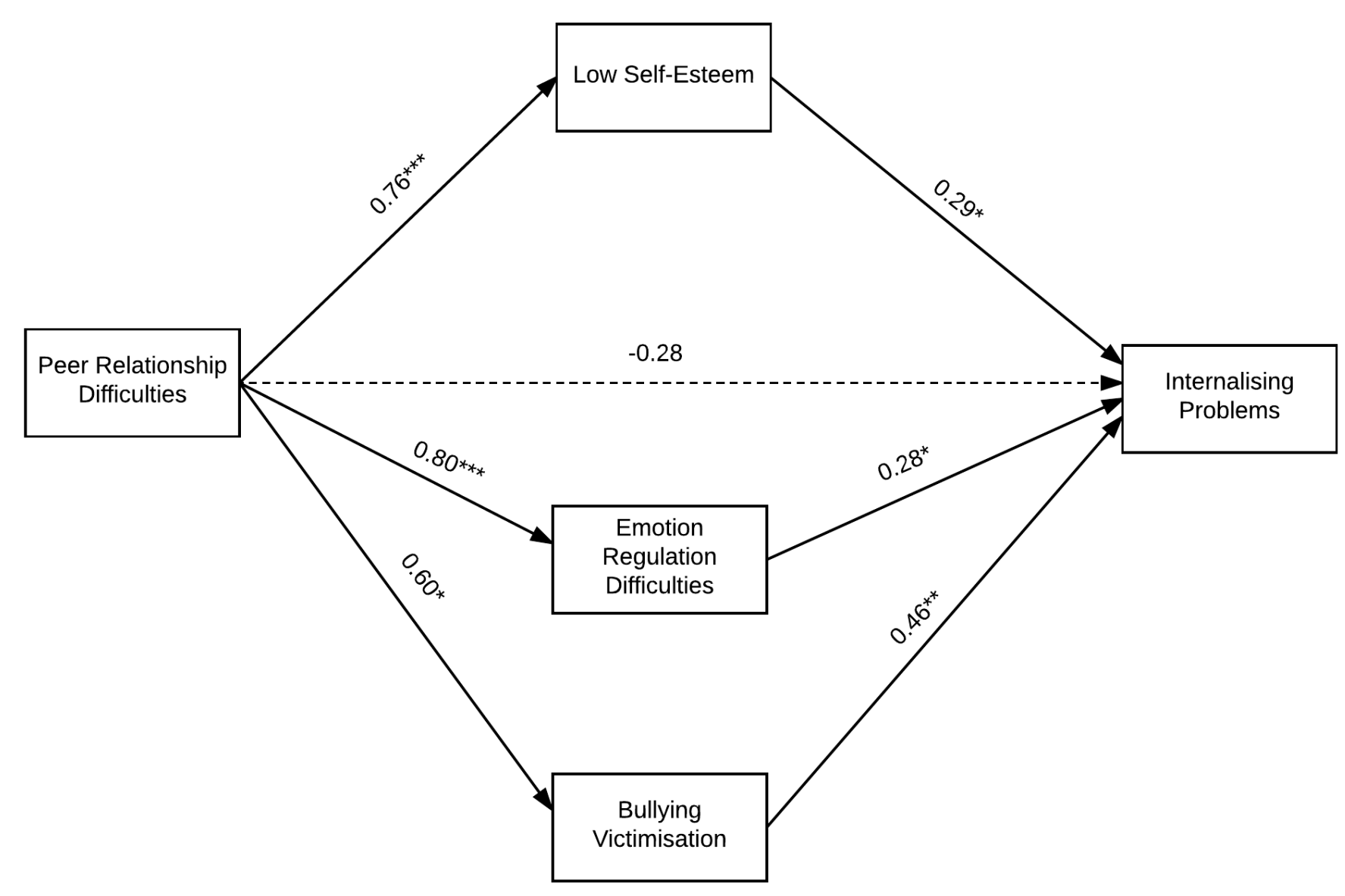

